\title{
Interplay between resonant tunneling and spin precession oscillations in all-electric all-semiconductor spin transistors
}

\author{
M. I. Alomar, Llorenç Serra, and David Sánchez \\ Institut de Física Interdisciplinària i Sistemes Complexos IFISC (CSIC-UIB), E-07122 Palma de Mallorca, Spain \\ and Departament de Física, Universitat de les Illes Balears, E-07122 Palma de Mallorca, Spain \\ (Received 23 December 2015; revised manuscript received 2 June 2016; published 1 August 2016)
}

\begin{abstract}
We investigate the transmission properties of a spin transistor coupled to two quantum point contacts acting as a spin injector and detector. In the Fabry-Pérot regime, transport is mediated by quasibound states formed between tunnel barriers. Interestingly, the spin-orbit interaction of the Rashba type can be tuned in such a way that nonuniform spin-orbit fields can point along distinct directions at different points of the sample. We discuss both spin-conserving and spin-flipping transitions as the spin-orbit angle of orientation increases from parallel to antiparallel configurations. Spin precession oscillations are clearly seen as a function of the length of the central channel. Remarkably, we find that these oscillations combine with the Fabry-Pérot motion, giving rise to quasiperiodic transmissions in the purely one-dimensional case. Furthermore, we consider the more realistic case of a finite width in the transverse direction and find that the coherent oscillations become deteriorated for moderate values of the spin-orbit strength. Our results then determine the precise role of the spin-orbit intersubband coupling potential in the Fabry-Pérot-Datta-Das intermixed oscillations.
\end{abstract}

DOI: 10.1103/PhysRevB.94.075402

\section{INTRODUCTION}

Spin transistors operate under the action of a spin-orbitcoupling potential that rotates the electronic spin traveling along a narrow channel [1]. Semiconductor heterostructures offer the possibility of generating spin-orbit interactions due to inversion asymmetry (Rashba type [2]), thus rendering semiconductor spintronics a rewarding area for spin information processing applications $[3,4]$. Importantly, the strength of the spin-orbit coupling can be tuned with an external electric field $[5,6]$, which provides the necessary gate tuning of the transistor switching mechanism. The last ingredient is the ability to both inject and detect spin-polarized currents. This can be done by attaching ferromagnetic terminals to the semiconductor channel. Yet a series conductivity mismatch owing to unequal Fermi wave vectors can hamper the system functionality [7-9]. Although spin-precession oscillations have been detected in ferromagnetic-semiconductor junctions [10] employing nonlocal voltage detection [11], the spin-injection efficiency between dissimilar materials tends to be low. The system performance can also be affected due to the presence of multiple channels [12-14], additional rotation of the spin of the traversing electron induced by intersubband coupling [15], the destructive effect of spin decoherence [16-18], the influence of gating [19,20], and the fact that the system can behave as a two-dimensional spin transistor [21-25].

An interesting alternative has very recently been put forward by Chuang et al. [26]. A pair of quantum point contacts (QPCs) works as spin injectors and detectors [27,28]. The electric confinement in the point constrictions leads to an effective magnetic field that polarizes the electrons in directions perpendicular to the spin-orbit field present in the central channel. As a consequence, the detector voltage becomes an oscillatory function of the middle gate voltage applied to the two-dimensional electron gas. Importantly, the system is fully nonmagnetic (neither ferromagnetic contacts nor external magnetic fields are needed for the operation principle) and relies on a semiconductor-only structure. This is an appealing feature that has been pursued in different proposals [29-34].

Consider the case when the conductance of both quantum point contacts is set below the value corresponding to a fully open mode. Then, the waveguide potentials can be described as tunnel barriers and transport across them occurs via evanescent states [35,36]. Effectively, the system electronic potential is globally seen as a double barrier with a quantum well of variable depth. It is well known that these potential landscapes in general support the presence of resonant scattering due to Fabry-Pérot-like oscillations arising from wave interference between the tunnel barriers. But at the same time we have spin-orbit-induced oscillations due to the precession of spins traveling between the barriers. Therefore, one would naturally expect a competition between resonant tunneling and spinprecession oscillations in a system comprising two serially coupled QPCs. Below, we show that this is indeed the case and that the combination of both oscillation modes leads to rich physics not only in the strictly one-dimensional case but also when more realistic samples with a finite transversal width are studied.

The subject of resonant tunneling effects and spin-orbit fields has been investigated in a number of works, giving rise to interesting predictions. For instance, Voskoboynikov et al. find that the transmission probability significantly changes in the presence of the Rashba coupling [37], while de Andrada e Silva and La Rocca obtain spin polarizations for an unpolarized beam of electrons impinging on a double-barrier nanostructure [38]. Koga et al. analyze spin-filter effects in triple-barrier diodes [39], whereas Ting and Cartoixà examine the doublebarrier case [40]. The dependence of the electronic tunneling on the spin orientation is treated by Glazov et al. [41]. These structures suffer from phase-breaking effects, as shown by Isić et al. [42].

In our work, we consider a purely ballistic system. Scattering is elastic, and the transmission probabilities are determined within the quantum scattering approach. Scattering can take place at the interfaces between the quantum point contacts 
and the quantum well or due to interaction between the spins and the spin-orbit interaction. Importantly and in contrast to previous works investigating spin-transistor transport properties, the spin-dependent transmission depends on the relative angle between the spin-orbit fields in the QPCs. This is an excellent property that allows us to tune the spin direction of the electrons impinging on the quantum well [26]. For a null relative angle, within a purely one-dimensional model we find that whereas the spin-conserving transmission shows resonant tunneling peaks as a function of the spin-orbit strength the spin-flip transmission always vanishes. Furthermore, for both types of transmissions the spin-precession oscillations as a function of the spin-orbit strength in the quantum well appear only when the QPCs have effective spin-orbit magnetic fields with an angle that differs from the spin-orbit coupling in the well. This effect can be also seen when the quantum well length is varied. However, we point out that the QPCs have an additional effect as tunnel barriers that lead to Fabry-Pérot resonances which can compete with the Datta-Das oscillations in the transmission curves, yielding quasiperiodic patterns. Now, since a realistic sample has a finite width, we also consider a quasi-one-dimensional system, in which case the spin-orbit intersubband coupling potential must also be taken into account. Remarkably, we find that our results derived from the one-dimensional model are also observable in two dimensions for moderately low values of the spin-orbit strength. This implies that the oscillation interplay discussed here can be probed with today's experimental techniques.

The content of our paper is structured as follows. Section II describes the system under consideration in two dimensions: a semiconductor layer with two quantum point contacts in series and a spatially inhomogeneous spin-orbit interaction applied on the QPCs and central region. The strict one-dimensional limit is addressed in Sec. III, where we have a doublebarrier potential modeling the two QPCs. We determine the eigenenergies and eigenfunctions in each region, and using matching methods, we find the transmission probabilities for a fixed incident spin. We perform an analysis of the transmission oscillations as a function of the relative orientation between the QPC effective magnetic fields and the spin-orbit interaction in the well, the strength of the spin-orbit coupling, and the width of the middle cavity. We stress that, depending on the direction of the spin polarization in the QPC regions, the transitions are dominated by processes that conserve or flip the spin direction. We also observe the combined effect of Datta-Das and Fabry-Pérot oscillations and obtain their characteristic frequencies. We find that modifying the strength of the spin-orbit coupling and the width of the central region, we can control the transmission probability for each spin. Section IV contains our analysis of the quasi-one-dimensional case. This discussion is important because it quantifies the role of spin-orbit intersubband coupling effects in both the Fabry-Pérot and Datta-Das oscillation modes. Finally, our conclusions are summarized in Sec. V.

\section{THEORETICAL MODEL}

We consider a semiconductor layer partitioned into five different regions as in Fig. 1: two reservoirs, two QPCs, and a quantum well $(\mathrm{QW})$. The blue areas are gate electrodes that

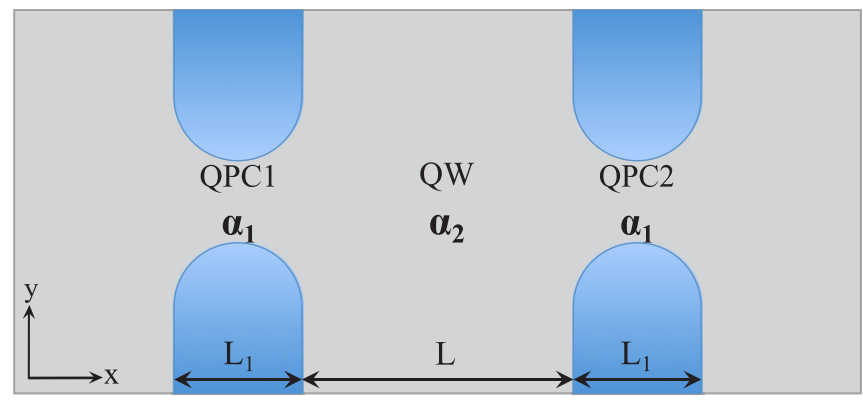

FIG. 1. Pictorial representation of our system. A semiconductor layer (light gray) with metallic electrodes (blue) shows two quantum point contacts in a series (QPC1 and QPC2) and a two-dimensional cavity in between $(\mathrm{QW})$. The spin-orbit coupling differs in each area $\left(\alpha_{1}\right.$ and $\left.\alpha_{2}\right)$ due to distinct electric fields applied to the electrodes (lateral in the metallic electrodes, perpendicular in the QW). $L_{1}$ and $L$ correspond to the width of QPCs and central region, respectively.

form constrictions in QPC1 and QPC2 between the left and right reservoirs and the central well. We take $x$ as the transport direction. The spin-orbit potentials acting on the QPCs (both with strength $\alpha_{1}$ ) and the QW (strength $\alpha_{2}$ ) are, in general, different [26]. Thus, our Hamiltonian reads

$$
\begin{gathered}
\mathcal{H}=\mathcal{H}_{0}+\mathcal{H}_{S O 1}+\mathcal{H}_{S O 2}, \\
\mathcal{H}_{0}=\frac{p_{x}^{2}+p_{y}^{2}}{2 m_{0}}+V(x, y), \\
\mathcal{H}_{S O 1}=\frac{\alpha_{1}}{\hbar}\left[(\vec{\sigma} \times \vec{p})_{z} \cos \phi+(\vec{\sigma} \times \vec{p})_{y} \sin \phi\right], \\
\mathcal{H}_{S O 2}=\frac{\alpha_{2}}{\hbar}(\vec{\sigma} \times \vec{p})_{z},
\end{gathered}
$$

where $\mathcal{H}_{0}$ represents the free part of the total Hamiltonian $\mathcal{H}$, with $p_{i}=-i \hbar \partial / \partial_{i}(i=x, y)$ being the linear momentum operator and $m_{0}$ being the conduction-band effective mass of the electrons in the semiconductor heterostructure. $V(x, y)$ confines electrons in the (transversal) $y$ direction and includes in $x$ two identical constrictions that define an intermediate region (the cavity or well) of length $L$. The spin-orbit terms of $\mathcal{H}$ are $\mathcal{H}_{S O 1}$ and $\mathcal{H}_{S O 2}$, where the first (second) is active on only the QPCs $(\mathrm{QW})$. Here, $\vec{\sigma}=\left(\sigma_{x}, \sigma_{y}, \sigma_{z}\right)$ and $\vec{p}=\left(p_{x}, p_{y}, 0\right)$ are the Pauli matrices and the momentum vector, respectively. In the central region, the $\alpha_{2}$ spin-orbit field [Eq. (4)] arises from the confining electric field perpendicular to the QW plane (the $z$ direction). In the constrictions, there exists in the $\alpha_{1}$ spin-orbit potential [Eq. (3)] an additional contribution from the lateral electric field applied to the QPCs along y. This field couples asymmetrically to the electrodes in Fig. 1 (blue areas), and as a consequence, a high spin-orbit interaction emerges in the QPCs, as experimentally demonstrated in Refs. $[26,27]$. The spin-orbit strength can be further enhanced by electron-electron interactions, doping potentials, or exchange correlations $[43,44]$. Our goal is not to describe these effects microscopically but rather focus on the transport properties. Hence, we lump these effects into the parameter $\alpha_{1}$, which can be tuned with the lateral electric field [27].

A convenient way of quantifying the strength of the two different components present in the QPCs (due to either lateral or perpendicular electric fields) is with the definition in Eq. (3) of the angle $\phi$. Therefore, we can turn off the 
lateral contribution by setting $\phi=0$, in which case $\mathcal{H}_{S O 1}$ and $\mathcal{H}_{\mathrm{SO} 2}$ are identical except for the spin-orbit strength. For $\phi=\pi / 2$ the lateral electric-field contribution to the spin-orbit potential dominates over that of the perpendicular electric field. Thus, the ensuing spin-orbit field in $\mathcal{H}_{S O 1}$ is orthogonal to that in $\mathcal{H}_{\mathrm{SO} 2}$. This ability to manipulate the orientation of the spin-orbit fields is crucial for the working principle of our system and has been proven in the experiments reported earlier [26]. It is a property that makes this device unique and that is absent in previous spin transistor studies. Another advantage of the QPCs is to reduce the wave-vector spread of injected electrons in contrast to extended interfaces [29]. Spin injection and detection with QPCs have been discussed in Refs. $[45,46]$ in the context of ballistic spin resonance. Here, we do not consider any external magnetic field, and all the spin dynamics originates from the effective magnetic fields due to the spin-orbit interactions present in the system, which makes our system an all-electric spin transistor.

\section{ONE-DIMENSIONAL CASE}

Let us, for the moment, disregard transverse channel effects and consider a purely one-dimensional model. We expect that this is a good approximation when the point contacts support only evanescent states. We will later discuss the more realistic case where the electronic waveguides have a nonzero transversal width. In this limit we describe the QPCs electrostatic potential $V(x, y)$ with a double tunnel barrier of width $L_{1}$ and height $V_{0}$ and the in-between cavity with a quantum well of length $L$ and bottom aligned with that of the reservoirs energy bands (see the sketch in Fig. 2). We then set $p_{y}=0$ in Eq. (1). Since the potential is piecewise constant, the eigenstates of $\mathcal{H}$ are readily found for the five regions defined in Fig. 2:

$$
\begin{gathered}
\Psi_{\ell s}^{0}(x) \equiv \Psi_{\ell s}^{I}=\Psi_{\ell s}^{V}=\frac{1}{\sqrt{2}}\left(\begin{array}{c}
\sqrt{1+s \sin \phi} \\
-i s \sqrt{1-s \sin \phi}
\end{array}\right) e^{i k_{\ell}^{(0)} x} \\
\Psi_{\ell s}^{1}(x) \equiv \Psi_{\ell s}^{I I}=\Psi_{\ell s}^{I V}=\frac{1}{\sqrt{2}}\left(\begin{array}{c}
\sqrt{1+s \sin \phi} \\
-i s \sqrt{1-s \sin \phi}
\end{array}\right) e^{i k_{\ell s}^{(1)} x}, \\
\Psi_{\ell s}^{2}(x) \equiv \Psi_{\ell s}^{I I I}=\frac{1}{\sqrt{2}}\left(\begin{array}{c}
1 \\
-i s
\end{array}\right) e^{i k_{\ell s}^{(2)} x}
\end{gathered}
$$

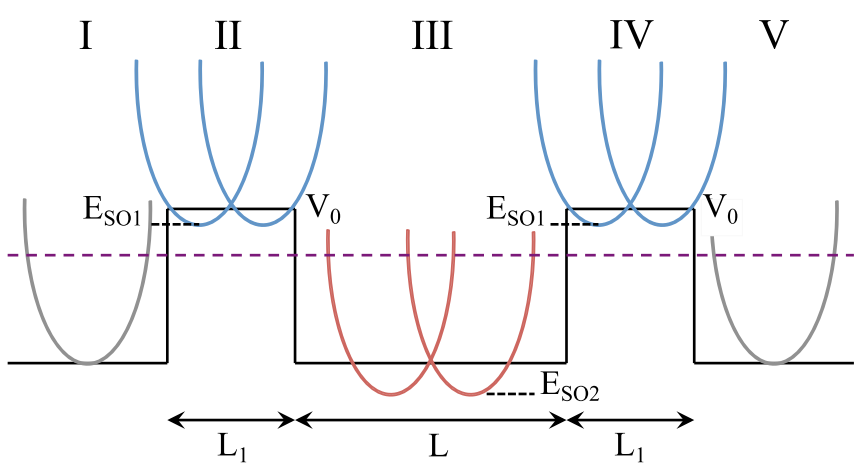

FIG. 2. Energy diagram of our system. The QPCs are described with barrier potentials of height $V_{0}$ and width $L_{1}$, whereas the size of the central region is denoted with $L$. We also plot the energy spectra in each region. Due to the spin-orbit coupling the band structure undergoes a spin splitting and an energy downshift $E_{S O}$. where $s= \pm$ is the spin index. For instance, $s=+$ corresponds to an electron with a spin pointing along $-y$ in the quantum well. We also label the states with the index $\ell= \pm$, which denotes the two possible momenta (i.e., the two possible wave-propagation directions) for fixed values of spin and energy $E$. The wave numbers read

$$
\begin{gathered}
k_{\ell}^{(0)} \equiv k_{\ell}^{I}=k_{\ell}^{V}=\ell \sqrt{\frac{2 m_{0}}{\hbar^{2}} E}, \\
k_{\ell s}^{(1)} \equiv k_{\ell s}^{I I}=k_{\ell s}^{I V}=\ell \sqrt{\frac{2 m_{0}}{\hbar^{2}}\left(E+E_{S O 1}-V_{0}\right)}-s k_{S O 1}, \\
k_{\ell s}^{(2)} \equiv k_{\ell s}^{I I I}=\ell \sqrt{\frac{2 m_{0}}{\hbar^{2}}\left(E+E_{S O 2}\right)}-s k_{S O 2},
\end{gathered}
$$

with $E_{S O i}=m_{0} \alpha_{i}^{2} /\left(2 \hbar^{2}\right)(i=1,2)$ being the downshift of the energy spectra due to the spin-orbit coupling, which also causes a horizontal band splitting $\Delta k$ characterized by the momentum $k_{S O i}=m_{0} \alpha_{i} / \hbar^{2}$. Equations (8), (9), and (10) depend on the energy of the incident electrons, which in the following we set equal to the Fermi energy $E_{F}$. Finally, we observe that both Eqs. (5) and (6) have the same spinor. Since the spin-quantization axis in the reservoirs is not fixed, we select it parallel to the spin direction on the adjacent QPCs.

We are now in a position to solve the scattering problem in Fig. 2. We focus on the case $0<E<V_{0}-E_{S O 1}$. This indicates that we are working with evanescent states in the QPC regions (II and IV). Hence, $k_{\ell s}^{(1)}$ acquires an imaginary part but generally also possesses a real part. We emphasize that this differs from the case of tunnel barriers without spin-orbit coupling [35]. On the other hand, both $k_{\ell s}^{(0)}$ and $k_{\ell s}^{(2)}$ are always real numbers. The matching method allows us to determine all reflection and transmission amplitudes for an incoming electron, which we take as impinging from the left. The matching conditions are

$$
\begin{gathered}
\Psi(\epsilon)-\Psi(-\epsilon)=0, \\
\Psi^{\prime}(\epsilon)-\Psi^{\prime}(-\epsilon) \\
=\frac{-i m_{0}}{\hbar^{2}}\left\{-\left[\alpha_{2}(\epsilon)-\alpha_{2}(-\epsilon)\right] \sigma_{y}\right. \\
\left.\quad+\left[\alpha_{1}(\epsilon)-\alpha_{1}(-\epsilon)\right]\left(\sin \phi \sigma_{z}-\cos \phi \sigma_{y}\right)\right\} \Psi(\epsilon),
\end{gathered}
$$

where $\epsilon$ is an infinitesimal quantity around each interface. Equation (11) is a statement of wave-function continuity. Equation (12) is derived from imposing flux conservation [47]. Notice that in the absence of spin-orbit interaction we recover the condition of continuity for the wave-function derivative. In the presence of spin-orbit coupling, this condition must be generalized according to Eq. (12).

Since transport is elastic, energy is conserved, and the transmission $T^{s^{\prime} s}$ and reflection $R^{s^{\prime} s}$ probabilities depend on a given $E$. However, spin can be mixed after scattering, and an incident electron with spin $s$ is reflected or transmitted with spin $s^{\prime}$. First, we analyze in Fig. 3 the main properties of $T^{s^{\prime} s}$ and $R^{s^{\prime} s}$ when we change the relative orientation between the QPCs and the QW spin-orbit fields. We choose the strength of the interaction in the QPCs $\left(\alpha_{1}\right)$ and in the QW $\left(\alpha_{2}\right)$ from Ref. [26]. We tune $\phi$ from zero (spins parallel oriented along the system) to $\pi / 2$ (spin axes perpendicularly oriented). In 


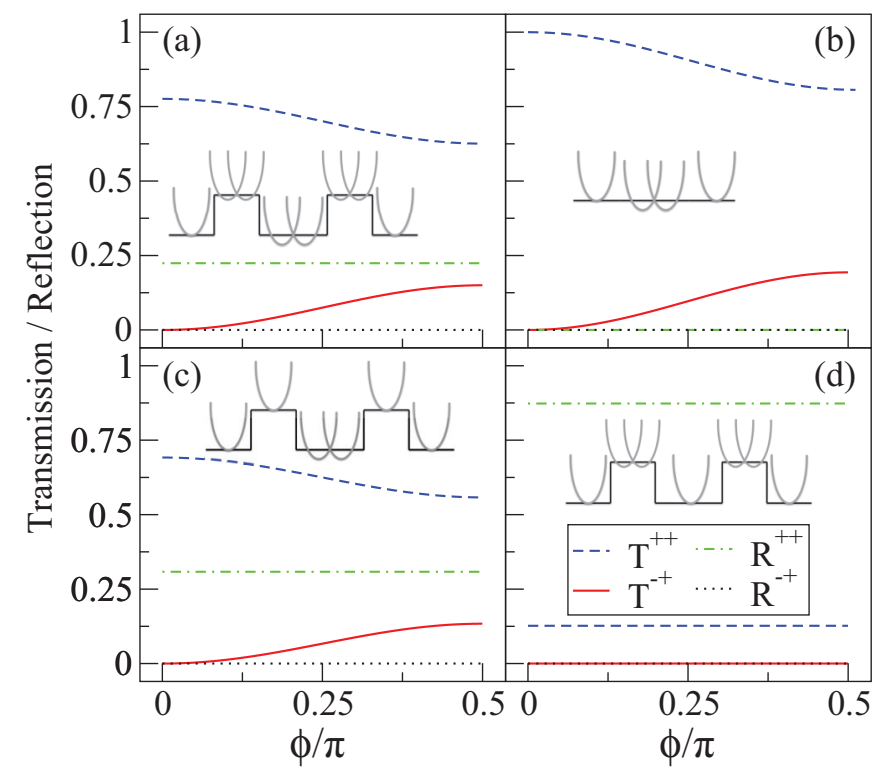

FIG. 3. Transmission and reflection probabilities as a function of the relative angle $\phi$ between spin-orbit fields in the QPC and the QW. $T^{s^{\prime} s}\left(R^{s^{\prime} s}\right)$ in the transmission (reflection) probability from an electronic state of spin $s= \pm$ to spin $s^{\prime}= \pm$ along the $-y$ direction. Parameters in (a) are $\alpha_{1}=20.16 \mathrm{meV} \mathrm{nm}, \alpha_{2}=25.18 \mathrm{meV} \mathrm{nm}$, $L=440.83 \mathrm{~nm}, L_{1}=28.02 \mathrm{~nm}, V_{0}=4.94 \mathrm{meV}$, and $E_{F}=4 \mathrm{meV}$. In (b) we remove the tunnel barriers $\left(L_{1}=0\right)$. In (c) [(d)] we cancel the spin-orbit interaction in the QPCs (QW): $\alpha_{1}=0\left(\alpha_{2}=0\right)$.

Fig. 3(a) we observe that, independent of the value of $\phi$, the electrons are reflected in the same spin state as the incoming one and that the reflection probability is roughly constant as a function of $\phi$. We understand this effect as being due to the spin orientations of electrons in regions I and II of Fig. 2, which are the same. In contrast, the transmission probability has both spin contributions for all values of $\phi$ except for the parallel configuration, for which $T^{-+}=0$ since there exists no spin polarization. We also remark that as $\phi$ increases, i.e., as the injected spin direction is rotated from $-y$ to $z$, $T^{-+}$increases while $T^{++}$decreases since for higher $\phi$ the perpendicular component of the spin direction becomes larger and its contribution to the transmission thus increases.

Let us further clarify the effects discussed above considering a few special cases. If we make $L_{1}=0$ (no tunnel barriers), the reflection probability is trivially zero [see Fig. 3(b)], and the transmission functions follow the same behavior as in Fig. 3(a) for which $L_{1}$ is nonzero. In Fig. 3(c) we observe that if we turn off the spin-orbit coupling on the QPCs $\left(\alpha_{1}=0\right)$, the transmission decreases compared with the values in Fig. 3(a). As a consequence, we infer that the spin-orbit coupling enhances the transmission properties of our double-barrier system. This may seem counterintuitive: when the spin-orbit interaction is present, one would naively expect more scattering and smaller transmission. However, we stress that the spin-orbit coupling lowers the energy-band bottom of the barrier, thus amplifying the role of the evanescent states (their characteristic decay length increases) and reducing consequently the reflection probability. Finally, when we take $\alpha_{2}=0$ (no spin-orbit interaction in the quantum well), all transport coefficients become independent of the angle $\phi$ [Fig. 3(d)] since the spin orientation in the central region is fixed. Furthermore, the reflection becomes higher due to the particular energy value, which lies around a resonance valley (see below).

Before proceeding, we notice that the case $\phi=0$ can be considerably simplified. The second term on the right-hand side of Eq. (3) cancels out, and we can write the projection of the Schrödinger equation $(\mathcal{H}-E) \Psi=0$ onto the spinor pointing along the $-y$ direction as

$$
\left[-\frac{\hbar^{2}}{2 m_{0}} \frac{d^{2}}{d x^{2}}-i s\left(\alpha_{1}+\alpha_{2}\right) \frac{d}{d x}+V_{0}-E\right] \Psi_{s}(x)=0,
$$

where $\alpha_{1}$ and $V_{0}$ are nonzero in regions II and IV, whereas $\alpha_{2}$ is nonvanishing in only region III (Fig. 2). Now, if we apply an appropriate gauge transformation $\Psi_{s}(x)=$ $\Psi(x) \exp \left[-i s \frac{m_{0}}{\hbar^{2}} \int d x^{\prime}\left(\alpha_{1}+\alpha_{2}\right)\right]$, we can recast Eq. (13) as

$$
\left(-\frac{\hbar^{2}}{2 m_{0}} \frac{d^{2}}{d x^{2}}+V_{1}-V_{2}-E\right) \Psi(x)=0,
$$

which is independent of the spin. Here, $V_{1}=V_{0}-E_{S O 1}$ in regions II and IV and is zero otherwise, while $V_{2}=E_{S O 2}$ in region III. This potential corresponds to a double barrier of renormalized height $V_{1}$ and a quantum well of depth $V_{2}$ in the central region. Clearly, the spin-orbit coupling effectively lowers the top of the barrier potential, as discussed earlier. Solving the scattering problem, we obtain a resonant condition that depends on all the parameters of our system,

$$
k_{\ell s}^{(2)} L=n \pi+f\left(\alpha_{1}, \alpha_{2}, L_{1}\right),
$$

where $k_{\ell s}^{(2)}$ is the wave number in the central region [Eq. (10)], $n=1,2, \ldots$ labels the different resonances, and $f\left(\alpha_{1}, \alpha_{2}, L_{1}\right)$ is a complicated function of $\alpha_{1}, \alpha_{2}$, and $L_{1}$ but independent of the QW length. The condition given by Eq. (15) can be numerically shown to hold also for the general case $\phi \neq 0$. However, in this case spin-precession effects must also be taken into account.

Figure 4 shows how our system reacts to changes applied to the spin-orbit strength in the central region $\alpha_{2}$. The parallel configuration $(\phi=0)$ is plotted in Fig. 4(a), where we observe resonance peaks for certain values of spin-orbit interaction and a fixed Fermi energy. As the spin-orbit coupling increases, the quantum well becomes deeper, and as a consequence, there appear new quasibound states between the two barriers that fulfill Eq. (15). When the energy of the incident electron hits one of these states, the transmission probability is maximal. Therefore, the spin-orbit interaction acts in our system as a gate voltage by shifting the resonances of the quantum well [48]. Our system then behaves as an analog of a Fabry-Pérot resonator tuned with a spin-orbit potential. Note that the resonances appear for only $T^{++}$since for $\phi=0$ the spins are parallel and one always obtains $T^{-+}=0$. This can be better understood if we take $L_{1}=0$, in which case the double-barrier potential disappears, and we obtain an almost transparent system independent of the depth of the quantum well [Fig. 4(b)]. Here, the energy of the electron is sufficiently high that its wave is mostly unaffected by the well discontinuity. Only for strong enough spin-orbit strengths does the transmission show weak oscillations (Ramsauer effect). We 


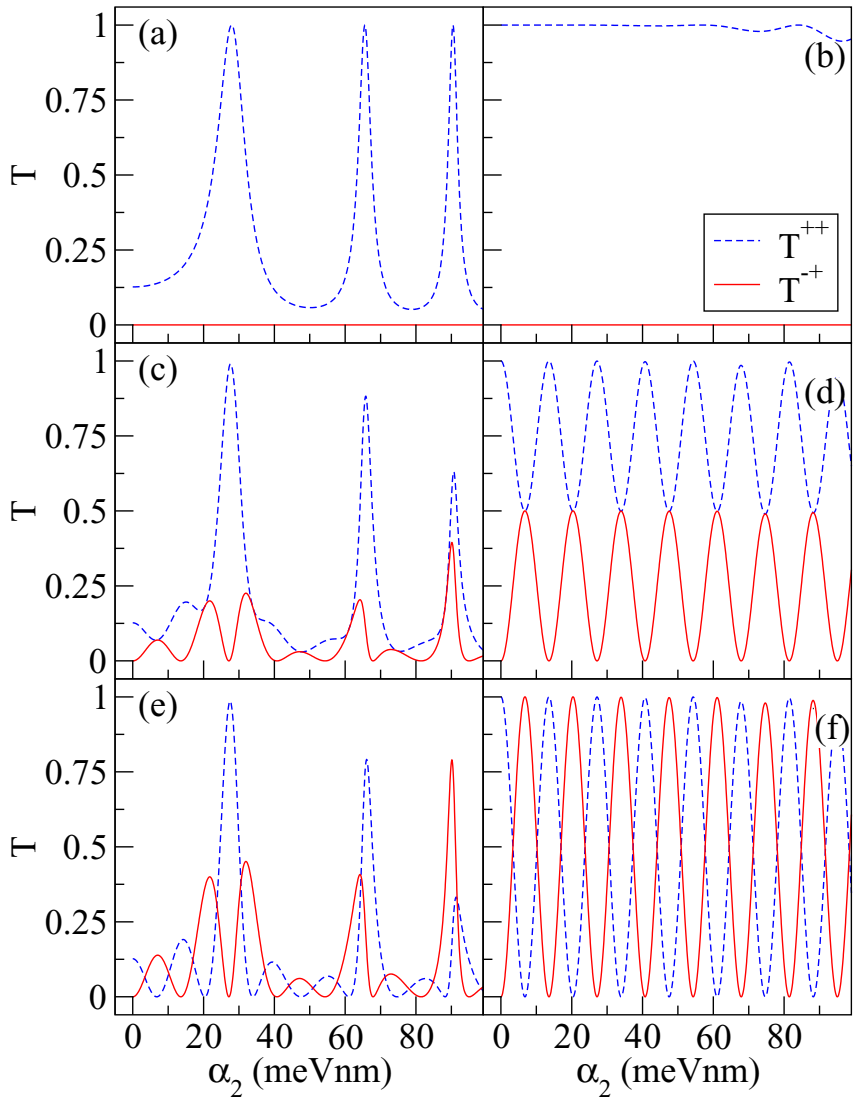

FIG. 4. Transmission probabilities as a function of the spin-orbit strength in the central region $\alpha_{2}$ for $\alpha_{1}=20.16 \mathrm{meV} \mathrm{nm}, L=$ $440.8 \mathrm{~nm}, V_{0}=4.94 \mathrm{meV}$, and $E_{F}=4 \mathrm{meV}$. The left panels have $L_{1}=28.02 \mathrm{~nm}$, while the right panels have $L_{1}=0$. The orientation angle is varied from top to bottom: $\phi=0$ for (a) and (b), $\phi=\pi / 4$ in (c) and (d), $\phi=\pi / 2$ for (e) and (f).

also find that the off-diagonal transmission coefficient is zero. This originates from the fact that in the parallel configuration the spin cannot be flipped, in agreement with the case $\phi=0$ in Fig. 3(d).

In Figs. 4(c) and 4(d) we take $\phi=\pi / 4$; that is, the wave is spin polarized $45^{\circ}$ with respect to $-y$. Let us first eliminate the double-barrier potential $\left(L_{1}=0\right)$ and focus on the effects from only the central region [see Fig. 4(d)]. We observe that both $T^{++}$and $T^{-+}$are nonzero and oscillate out of phase. These oscillations are a consequence of the spin-transistor effect predicted by Datta and Das [1]. We find $T^{++}=1$ and $T^{-+}=0$ for $\alpha_{2}=0$, but then both transmissions become modulated as we increase the spin-orbit strength since the QW energy bands show a larger spin splitting $\Delta k=m_{0} \alpha_{2} / \hbar^{2}$. For certain values of $\alpha_{2}, T^{++}\left(T^{-+}\right)$attains its minimum (maximum) value of 0.5 . Importantly, the nature of these transmission oscillations fundamentally differs from the resonances in Fig. 4(a). To see this, we next obtain the spin-precession frequency from the relation [1]

$$
T^{++} \propto \cos ^{2}(\Delta k L) .
$$

This expression implies that the maximum condition is reached at $\Delta k L=n^{\prime} \pi\left(n^{\prime}=1,2, \ldots\right)$. For the parameters in Fig. 4(d) this corresponds to $\alpha_{2} \simeq 13.6 n^{\prime} \mathrm{meV} \mathrm{nm}$.

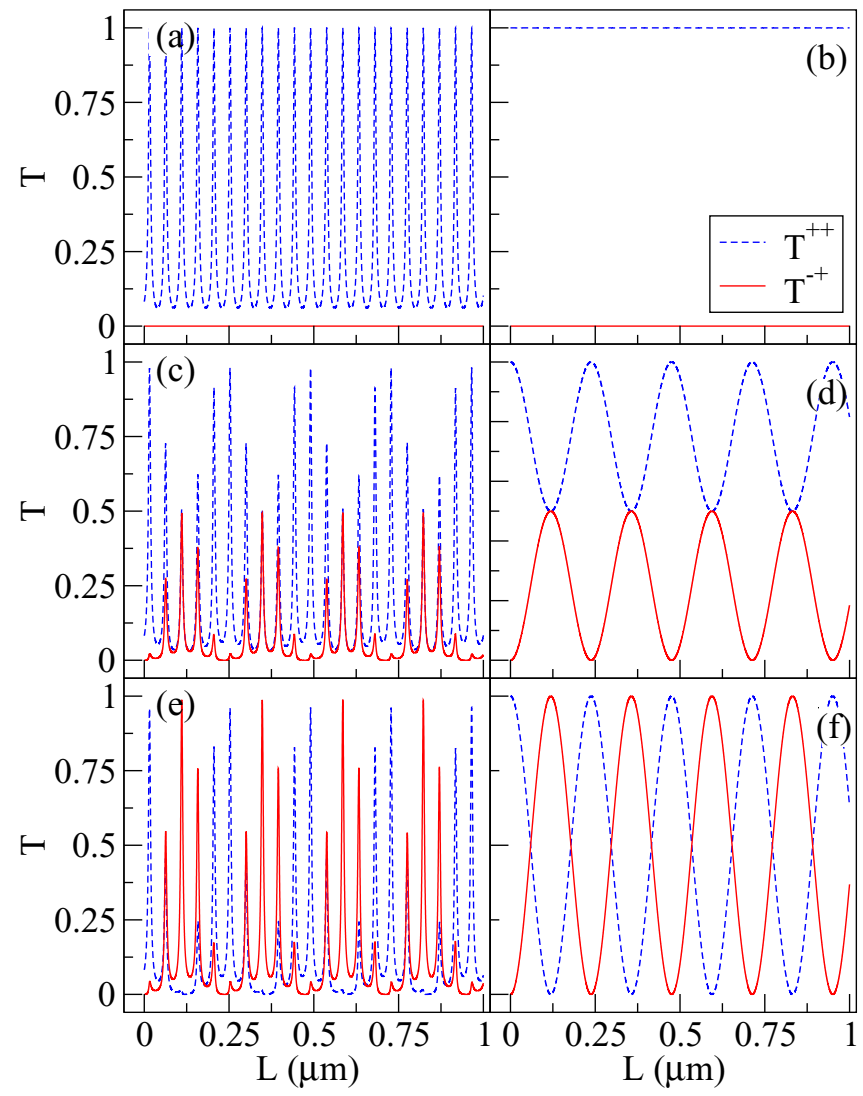

FIG. 5. Transmission probabilities as a function of the central region width $L$ for $\alpha_{1}=20.16 \mathrm{meV} \mathrm{nm}, \alpha_{2}=25.18 \mathrm{meV} \mathrm{nm}, V_{0}=$ $4.94 \mathrm{meV}$, and $E_{F}=4 \mathrm{meV}$. The left panels have $L_{1}=28.02 \mathrm{~nm}$, while the right panels have $L_{1}=0$. The orientation angle is varied from top to bottom: $\phi=0$ for (a) and (b), $\phi=\pi / 4$ in (c) and (d), $\phi=\pi / 2$ for (e) and (f).

More interestingly, we now turn on the double-barrier potential and allow for the interplay between Fabry-Pérot and Datta-Das oscillations. The superposition of the two effects can be seen in Fig. 4(c). We observe that (i) the resonance peaks for $T^{++}$become somewhat quenched and (ii) the off-diagonal coefficient $T^{-+}$shows an irregular series of oscillating peaks. The effect is more intense in the perpendicular configuration $(\phi=\pi / 2)$ [see Fig. 4(e)]. Both transmissions oscillate now between 0 and 1 with opposite phases [Fig. 4(f)], and the combination of both types of oscillations yields the curves depicted in Fig. 4(e).

It is now natural to ask about the effect of tuning the QW length $L$. We show this in Fig. 5 for the same orientation angles as in Fig. 4 but fixing the spin-orbit strength $\alpha_{2}$. When $\phi=0$, Fig. 5(a) presents for $T^{++}$narrowly spaced oscillations since, as we increase the width of the central cavity, there appear more internal modes that, at fixed values of $L$, are resonant with the incident wave (Fabry-Pérot effect). The resonant condition from Eq. (15) implies that the transmission is peaked at $L \simeq(47.5 n+8.3) \mathrm{nm}(n=1,2, \ldots)$. For $\phi=0$ spin flipping is not possible, and $T^{-+}=0$. When the constrictions are turned off $\left(L_{1}=0\right)$, we have a completely open system, and the transmission stays constant at its maximum value [see Fig. 5(b)]. As we increase the spin orientation angle 
$[\phi=\pi / 4$ in Figs. 5(c) and 5(d) and $\phi=\pi / 2$ in Figs. 5(e) and 5(f)], the spin-transistor effect begins to contribute as we observe a spin precession for both $T^{++}$and $T^{-+}$, modulated by their characteristic frequency, namely, $L \simeq 237.6 n^{\prime} \mathrm{nm}$ ( $\left.n^{\prime}=1,2, \ldots\right)$. We find that when $L_{1}=0$ (no tunnel barriers), the Fabry-Pérot resonances disappear, and only the Datta-Das oscillations are present [Fig. 5(d) and 5(f)], as expected.

Remarkably, when both oscillation modes are present, we find that the transmission becomes quasiperiodic [Fig. 5(c) and 5(e)]. This effect arises from the combination of at least two oscillations whose characteristic frequencies are incommensurate [49]. In our system, the Fabry-Pérot frequency is given by $f_{F P}=\frac{1}{\pi} \sqrt{\frac{2 m_{0}}{\hbar^{2}} E+k_{S O 2}^{2}}$, whereas that of the spin precession motion is expressed as $f_{s p}=2 k_{S O 2} / \pi$. Clearly, its ratio $f_{F P} / f_{s p}$ is quite generally an irrational number. In related systems, quasiperiodic oscillations have been predicted to occur in double quantum dots with incommensurate capacitance couplings [50] and in ac-driven superlattices where the ratio between the ac frequency and the internal frequency is not a rational number [51]. Importantly, in our case the origin of both oscillations is purely quantum (wave interference and spin precession).

\section{QUASI-ONE-DIMENSIONAL CASE}

The above discussion demonstrates that two types of transmission oscillations can coexist in a double-barrier spinorbit-coupled resonant tunneling diode. However, the results were strictly limited to the one-dimensional (1D) case. We now consider the more realistic situation of a double QPC embedded in a quantum wire of finite width. The problem is not a mere extension that takes into account transverse channels since these channels become coupled via the Rashba intersubband mixing potential. This term causes spin-flip transitions between adjacent channels and generally destroys the spin-coherent oscillations [14]. Furthermore, it yields Fano line shapes [52] that dramatically alter the conductance curves [48,52-55]. We note that there exists another type of intersubband spin-orbit-coupling potential that occurs in coupled wells with two subbands [56]. Here, we consider the case of an intense confinement in the growth direction such that only the lowest subband is populated.

We consider the planar waveguide formed in a twodimensional (2D) electron gas lying on the $x-y$ plane as in Fig. 1. In the numerical simulations we consider a hard-wall confinement potential along $y$ and two square quantum point contacts in the $x$ direction. The system parameters are depicted in Fig. 6.

We take a given quantization axis $\hat{n}$ for the spin in the left and right contacts. The spin eigenfunctions are then denoted with $\chi_{s}(\eta)$, with $s= \pm$ being the eigenstate label and $\eta=\uparrow, \downarrow$ being the discrete variable. The full wave function $\Psi(x, y, \eta)$ is expanded in spin channels $\psi_{s}(x, y)$ as

$$
\Psi(x, y, \eta)=\sum_{s^{\prime}} \psi_{s^{\prime}}(x, y) \chi_{s^{\prime}}(\eta) .
$$

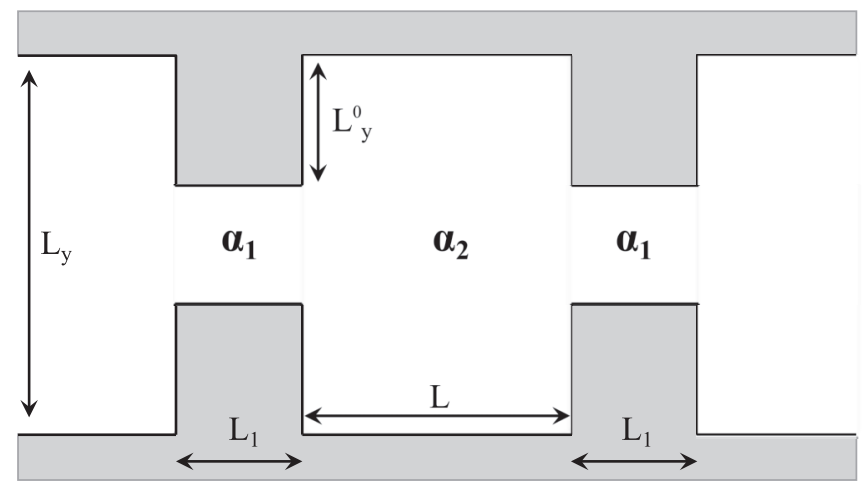

FIG. 6. Sketch of the double-quantum-point-contact system with a finite width $L_{y}$. Electrons can move in the white areas whereas forbidden regions are depicted in grey. The height of the constriction barriers is $L_{y}^{0}$. The rest of the parameters are defined as in the purely 1D case (Fig. 2).

Projecting the Schrödinger equation on the spin basis, we obtain coupled-channel equations,

$$
\begin{aligned}
& {\left[-\frac{\hbar^{2} \nabla^{2}}{2 m}+V(x, y)\right] \psi_{s}(x, y)} \\
& \quad-\frac{i \hbar}{2} \sum_{s^{\prime}}\left\langle s\left|\sigma_{y}\right| s^{\prime}\right\rangle\left(V_{A}(x) \frac{\partial}{\partial x}+\frac{\partial}{\partial x} V_{A}(x)\right) \psi_{s^{\prime}}(x, y) \\
& \quad-\frac{i \hbar}{2} \sum_{s^{\prime}}\left\langle s\left|\sigma_{z}\right| s^{\prime}\right\rangle\left(V_{B}(x) \frac{\partial}{\partial x}+\frac{\partial}{\partial x} V_{B}(x)\right) \psi_{s^{\prime}}(x, y) \\
& \quad+\frac{i \hbar}{2} \sum_{s^{\prime}}\left\langle s\left|\sigma_{x}\right| s^{\prime}\right\rangle V_{A}(x) \frac{\partial}{\partial y} \psi_{s^{\prime}}(x, y)
\end{aligned}
$$

where the potentials $V_{A}(x)$ and $V_{B}(x)$ are responsible for the coupling between the different spin channels $s= \pm$. In general, the Pauli-matrix elements in Eq. (18) depend on $\hat{n}$. To connect with the 1D case discussed in Sec. III we take $\hat{n}=-\hat{y}$, which makes the $\sigma_{y}$ term diagonal, but those with $\sigma_{x}$ and $\sigma_{z}$ remain nondiagonal. Coupling between opposite spin states is therefore always present in the quasi-1D case when $\left(V_{A}, V_{B}\right) \neq 0[57,58]$.

In Eq. (18) the potentials $V_{A}$ and $V_{B}$ read

$$
\begin{gathered}
V_{A}(x)=\alpha_{1} \cos \phi \mathcal{P}_{1}(x)+\alpha_{2} \mathcal{P}_{2}(x)+\alpha_{1} \cos \phi \mathcal{P}_{3}(x), \\
V_{B}(x)=-\alpha_{1} \sin \phi \mathcal{P}_{1}(x)-\alpha_{1} \sin \phi \mathcal{P}_{3}(x),
\end{gathered}
$$

where the projectors $\mathcal{P}_{i}(x)$ partition the $x$ domain in regions $i=1$ (left QPC), $i=2$ (QW), and $i=3$ (right QPC). These two potentials yield qualitatively different spin-flip couplings since $V_{B}$ only appears with $\partial / \partial x$, while $V_{A}$ appears with both $\partial / \partial x$ and $\partial / \partial y$. As before, $\phi$ is the angle defining the relative orientation of the Rashba fields. Notably, $V_{B}(x)$ vanishes with $\phi=0$, and then, for the quantization axis along $y$, the only spin-flip coupling in Eq. (18) is via the last term depending 

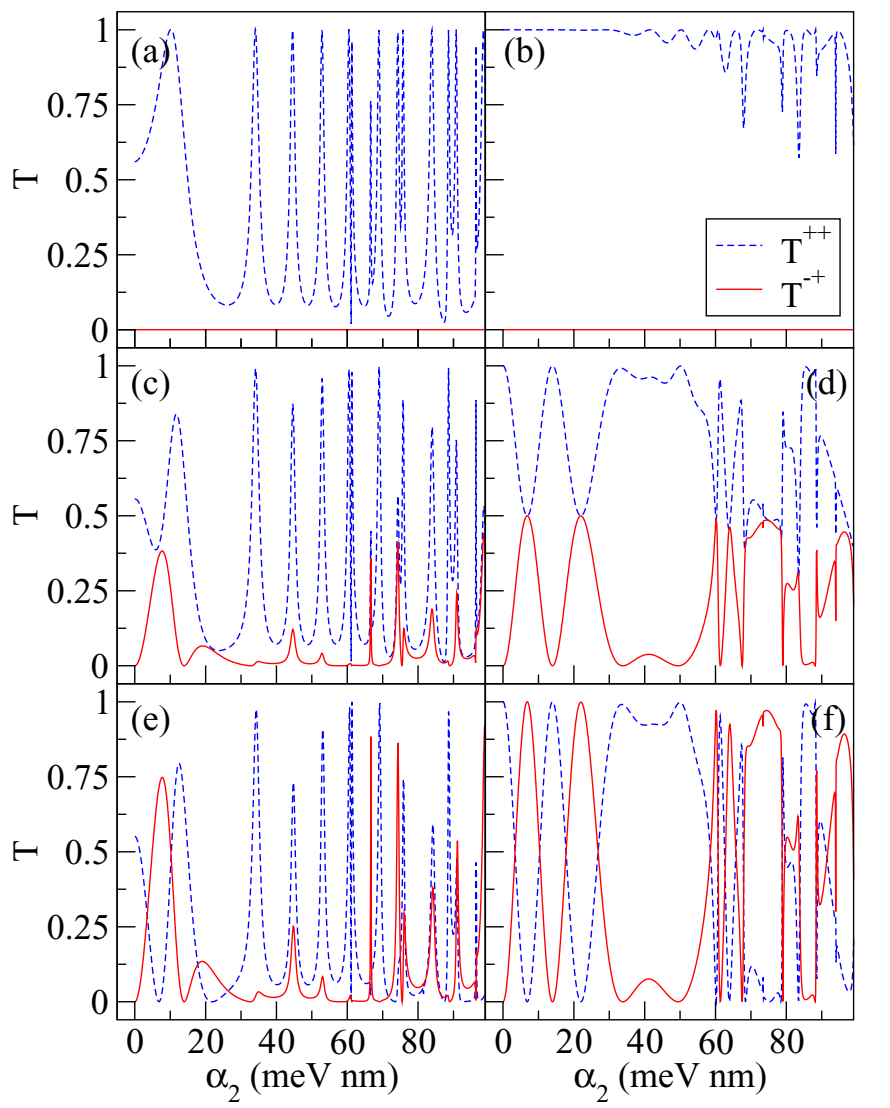

FIG. 7. Transmission probabilities for a quasi-one-dimensional double-quantum-point-contact system as a function of the spin-orbit strength in the central region $\alpha_{2}$. Parameters: $\alpha_{1}=20.16 \mathrm{meV} \mathrm{nm}$, $L=440.8 \mathrm{~nm}, L_{1}=10.91 \mathrm{~nm}, L_{y}^{0}=39.29 \mathrm{~nm}, L_{y}=87.29 \mathrm{~nm}$, and $E_{F}=4 \mathrm{meV}$. The left panels have $L_{1}=10.91 \mathrm{~nm}$, while the right panels have $L_{1}=0$. The orientation angle is varied from top to bottom: $\phi=0$ for (a) and (b), $\phi=\pi / 4$ in (c) and (d), $\phi=\pi / 2$ for (e) and (f).

on $\partial / \partial y$. To be effective, this spin-flip coupling requires that at least two transverse modes (differing in the nodes along $y$ ) are propagating in the asymptotic leads [52]. Otherwise, as we show below, there is no spin-flip when $\hat{n}$ lies along $y$.

Equation (18) is solved with the quantum-transmitting boundary method [59] on a uniform grid. The resulting transmission probability as a function of the middle spin-orbit strength $\alpha_{2}$ is shown in Fig. 7. We recall that the transmission is expressed in the $-y$-direction basis. Like in Fig. 4 we distinguish the case with the constrictions (left panels) from the case without the QPCs (right panels). For $\phi=0$ [Fig. 7(a)] we quench the spin-precession oscillations since the injected spins are parallel to the Rashba field. Then, the cross transmission $T^{-+}$vanishes identically. The resonant tunneling peaks qualitatively agree with the 1D case [see Fig. 4(a)]. Likewise, the Ramsauer oscillations that arise when the QPCs are absent [Fig. 7(b)] are visible at large values of $\alpha_{2}$ [see Fig. 4(b)]. The agreement in both cases is good for small values of $\alpha_{2}$. This is reasonable since Rashba intersubband coupling is negligible if $\alpha_{2} \ll \hbar^{2} / m L_{y}$ [1]. For larger $\alpha_{2}$ we observe in Fig. 7(b) sharp dips that originate from the Fano-Rashba effect [52] and that are unique to quasi-one-dimensional waveguides with nonuniform spin-orbit coupling as in our case. Strikingly enough, as $\alpha_{2}$ increases we detect in Fig. 7(a) more resonant peaks than in the strict $1 \mathrm{D}$ case. We explain this effect as follows. For $\alpha_{1}=\alpha_{2}=0$ the cavity works as a resonator with multiple resonances. If the cavity is closed, the bound levels can be described with a pair of natural numbers $\left(n_{1}, n_{2}\right)$ since its potential corresponds to a $2 \mathrm{D}$ infinite well [60]. To a good approximation, the electronic scattering when the cavity is open obeys a conservation law that fixes the transversal component of motion [61]. Accordingly, $n_{2}$ is conserved upon traversing the cavity, and the transmission shows less peaks than bound states in the closed cavity. In the presence of spin-orbit coupling, the conservation law does not have to hold and more resonances then emerge.

For $\phi=\pi / 4$ the injected electrons are spin rotated with regard to the $\alpha_{2}$ field, and spin-precession oscillations of the Datta-Das-type are expected. This can be more distinctly seen in Fig. 7(d), where the QPC widths are set to zero. Up to $\alpha_{2} \simeq 30 \mathrm{meV} \mathrm{nm}$ the oscillations are smooth as in Fig. 4(d). For larger $\alpha_{2}$ the subband mixing potential starts to play a significant role. As a consequence of the spin mixing induced by the $p_{y}$ term, the precession oscillations become irregular [14], and the transmission curves can no longer be determined by a single frequency. When combined with the Fabry-Pérot oscillations, the transmission line shapes are transformed into nonharmonic functions of $\alpha_{2}$ [see Fig. 7(c)], and our previous 1D analysis in terms of quasiperiodic oscillations does not hold. For completeness, we also show the case $\phi=\pi / 2$ for which the Data-Das frequency is higher (the spins are injected perpendicular to the Rashba field), but the spin oscillations turn out to be nonuniform as $\alpha_{2}$ grows, as illustrated in Fig. 7(f). The overall transmission curves [Fig. 7(e)] qualitatively follow the pattern observed in the case $\phi=\pi / 4$.

In Fig. 8 we analyze the dependence on the central-cavity width $L$. We set the spin-orbit strength $\alpha_{2}$ to a moderate value to highlight the effects due to the Rashba intersubband coupling term. Figure 8(a) shows the transmission for $L_{1}=0$ and $\phi=\pi / 2$. This implies that only oscillations from the spin dynamics are present since resonant tunneling effects are not allowed. Unlike Fig. 5(f), here the oscillations are not uniform for both transmission probabilities, $T^{++}$and $T^{-+}$. The Fabry-Pérot peaks are more regular, as shown in Fig. 8(b), where $L_{1}$ is nonzero and $\phi=0$ in order to forbid spin-precession oscillations. This suggests that the Rashba intersubband potential has a stronger impact on the Datta-Das oscillations than on the Fabry-Pérot peaks. In Fig. 5(c) we show characteristic transmission curves for nonzero $L_{1}$ and $\phi=\pi / 2$, in which case both oscillation modes come into play. Compared to the 1D case in Fig. 5(e), the oscillations are now more intricate: their amplitudes strongly fluctuate with increasing $L$, and their frequency cannot be described in terms of combinations of individual frequencies.

In order to complete the analysis of our system we present in Fig. 9 the transmission probability as a function of the Fermi energy for the same parameters as above. In Fig. 9(a) we consider the case without the QPCs $\left(L_{1}=0 \mathrm{~nm}\right)$ and apply a spin-orbit interaction in the central region such that its direction lies orthogonal to that of the leads $(\phi=\pi / 2)$. We find an approximate transmission quantization of $T=T^{++}+T^{-+}$ (black line) whenever a new propagating channel opens up as 

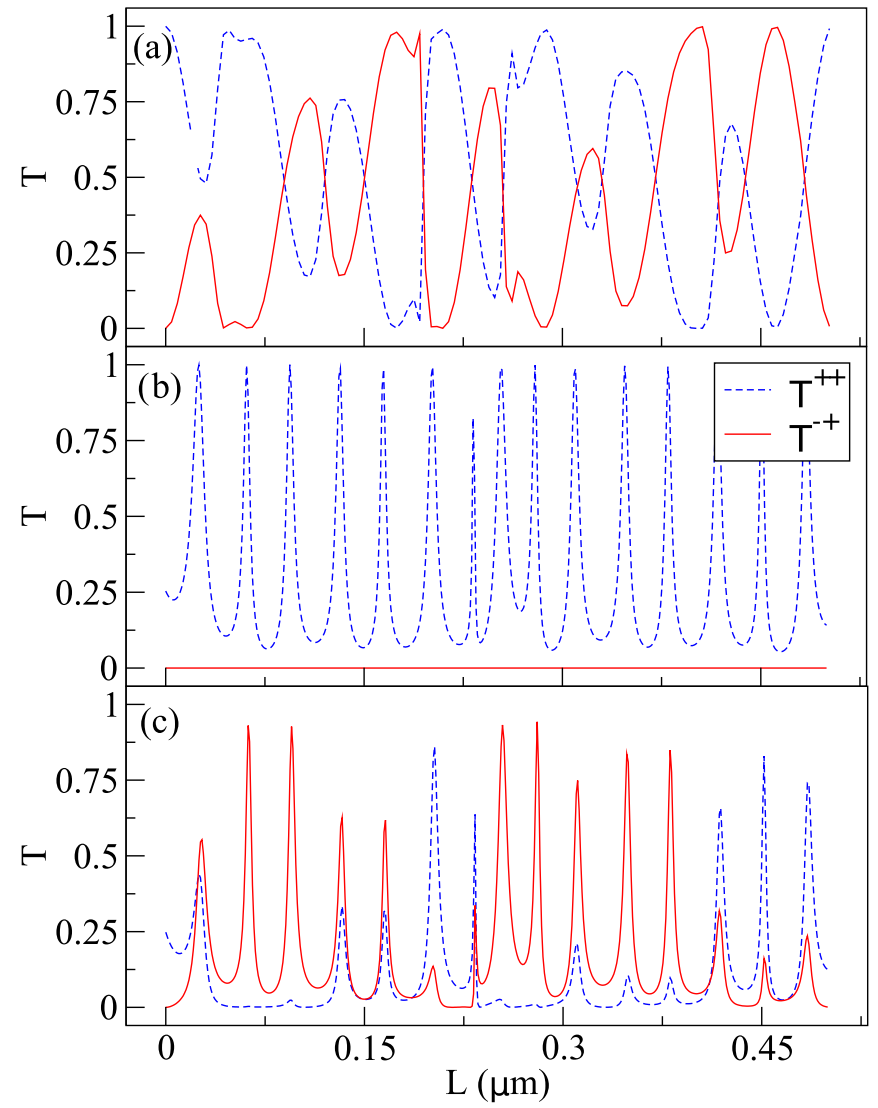

FIG. 8. Transmission probabilities for a quasi-one-dimensional double-quantum-point-contact system as a function of the width of the central region $L$. Parameters: $\alpha_{1}=20.16 \mathrm{meV} \mathrm{nm}, \alpha_{2}=65.47 \mathrm{meV}$ $\mathrm{nm}, L_{y}^{0}=39.29 \mathrm{~nm}, L_{y}=87.29 \mathrm{~nm}$, and $E_{F}=4 \mathrm{meV}$. Additionally, we set in (a) $L_{1}=0 \mathrm{~nm}$ and $\phi=\pi / 2$, in (b) $L_{1}=10.91 \mathrm{~nm}$ and $\phi=0$, and in (c) $L_{1}=10.91 \mathrm{~nm}$ and $\phi=\pi / 2$.

the Fermi energy surpasses the values $E_{n}=\hbar^{2} \pi^{2} n^{2} / 2 m_{0} L_{y}^{2}$ with $n=1,2, \ldots$ (recall that the confinement along the transverse direction is described with a hard-wall potential). We also observe in Fig. 9(a) the spin dependence due to the spin-orbit interaction in the middle region (solid red and dashed blue lines). The Fabry-Pérot peaks form when $\phi=0$ and $L_{1} \neq 0$ [see Fig. 9(b)]. Here, the transmission is zero until the Fermi energy is such that the first propagating state is allowed in the leads, which corresponds to $E_{F}>E_{1}=$ $1.23 \mathrm{meV}$. At the same time, in the QPCs we have evanescent states below the energy value $E_{1}^{Q P C}=4.93 \mathrm{meV}$. Then, the resonances ranging between these two energies are due only to tunneling transmission across the QPCs. The second channel in the leads opens up at $E_{2}=4.93 \mathrm{meV}$, but the transmission does not exceed 1 because we have just one open channel in the constrictions. When the third channel in the leads opens up, $E_{F}>E_{3}=11.10 \mathrm{meV}$, we observe dips in the diagonal transmission probability which correlate with peaks in the off-diagonal transmission. This effect originates from the coupling between propagating states in the system and quasibound states in the cavity. Finally, Fig. 9(c) shows the combination of Fabry-Pérot peaks and Datta-Das oscillations when the spin-orbit fields are perpendicular. Its behavior is similar to the Fabry-Pérot-Datta-Das oscillations discussed as
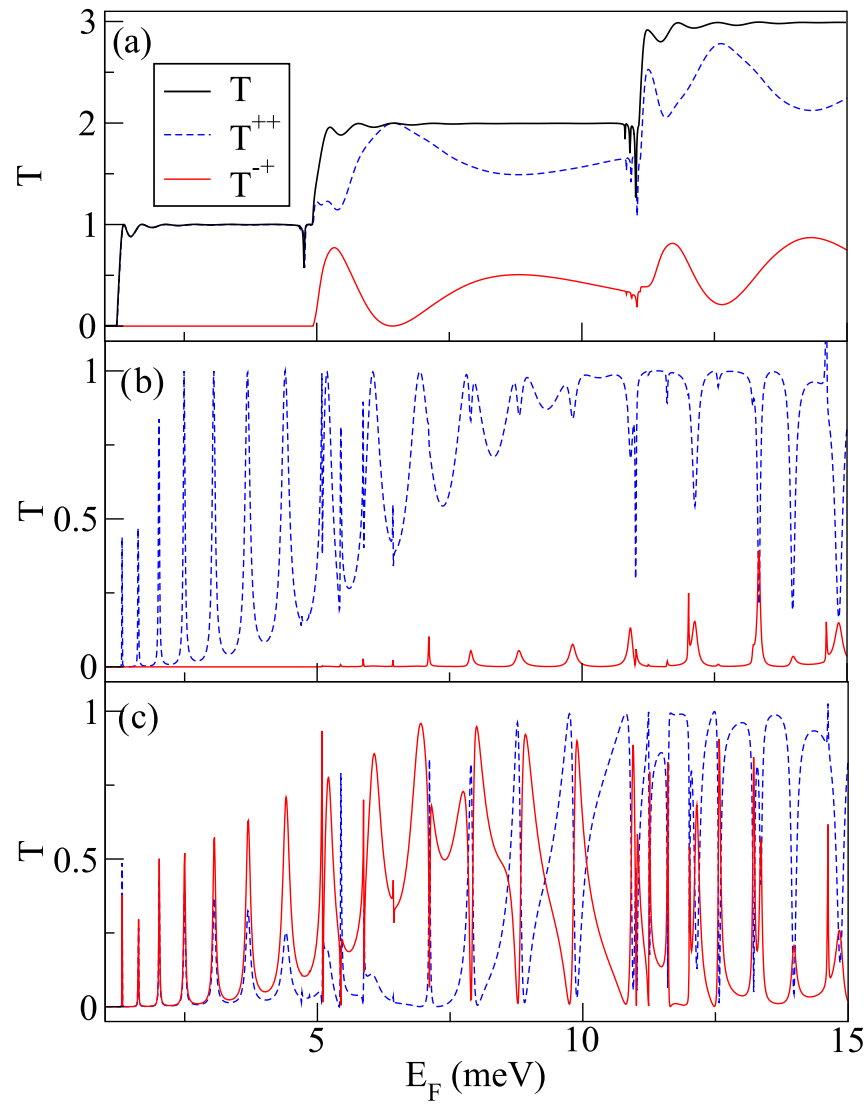

FIG. 9. Transmission probabilities for a quasi-one-dimensional double-quantum-point-contact system as a function of the position of the Fermi level $E_{F}$. Parameters: $\alpha_{1}=20.16 \mathrm{meV} \mathrm{nm}, \alpha_{2}=$ $65.47 \mathrm{meV} \mathrm{nm}, L_{y}^{0}=21.82 \mathrm{~nm}, L_{y}=87.29 \mathrm{~nm}$, and $L=440.8 \mathrm{~nm}$. Additionally, we set in (a) $L_{1}=0 \mathrm{~nm}$ and $\phi=\pi / 2$, in (b) $L_{1}=10.91$ $\mathrm{nm}$ and $\phi=0$, and in (c) $L_{1}=10.91 \mathrm{~nm}$ and $\phi=\pi / 2$.

a function of the spin-orbit coupling [Fig. 7(e)] and cavity length [Fig. 8(c)].

\section{CONCLUSIONS}

To sum up, we have investigated a spin-orbit quantum wire coupled to quantum point contacts. We have found that both resonant tunneling and spin-precession oscillations combine into complex patterns that can be explained with the aid of quasiperiodic modes in the strict $1 \mathrm{D}$ case. For the more realistic setup where the conducting channel has a finite width (2D case) we have discussed the important role of the Rashba intersubband coupling term as the spin-orbit strength increases.

We have used in our numerical simulations realistic parameters taken from the sample and measurements of Ref. [26]. Therefore, our predictions are within the realm of today's techniques. The angle between the spin-orbit fields in the QPCs and the quantum well can be tuned with lateral electric fields, while the spin-orbit strength can be manipulated with a gate terminal on top of the middle cavity. We have focused on the transmission, from which the two-terminal conductance $G$, which is experimentally accessible, readily follows in the zero-temperature limit. For finite temperatures we expect thermal smearing effects, but we have in mind low 
temperatures as in Ref. [26] (0.03 K). Thus, phonon effects can be safely neglected. Another detrimental effect would be the presence of disorder since we consider only ballistic systems and our predictions rely on quantum interference. Therefore, samples with large enough coherence length and mean free path would be needed, which are now routinely available [62]. Measurement of diagonal and off-diagonal conductances can be achieved, e.g., with ferromagnetic electrodes whose relative magnetization can be changed from parallel to antiparallel orientation in response to a small magnetic field [10]. The results regarding the length variation can be tested with different samples. Finally, the resolution of the conductance peaks would lie in the sub-meV range (see Fig. 9), which can be achieved by tuning an external back-gate electrode capacitively coupled to the sample.

Further extensions of our work could address high-field transport properties, in which case inelastic transitions in threedimensional resonant tunneling diodes can change the currentvoltage characteristics $[63,64]$. Another important issue for future works is the role of electron-electron interactions,

[1] S. Datta and B. Das, Electronic analog of the electro-optic modulator, Appl. Phys. Lett. 56, 665 (1990).

[2] E. I. Rashba, Properties of semiconductors with an extremum loop I. Cyclotron and combinational resonance in a magnetic field perpendicular to the plane of the loop, Sov. Phys. Solid State 2, 1109 (1960).

[3] J. Fabian, A. Matos-Abiague, C. Ertler, P. Stano, and I. Zutic, Semiconductor spintronics, Acta Phys. Slov. 57, 565 (2007).

[4] D. Bercioux and P. Lucignano, Quantum transport in Rashba spin-orbit materials: A review, Rep. Prog. Phys. 78, 106001 (2015).

[5] J. Nitta, T. Akazaki, H. Takayanagi, and T. Enoki, Gate Control of Spin-Orbit Interaction in an Inverted $\mathrm{In}_{0.53} \mathrm{Ga}_{0.47} \mathrm{As} / \mathrm{In}_{0.52} \mathrm{Al}_{0.48}$ As Heterostructure, Phys. Rev. Lett. 78, 1335 (1997).

[6] G. Engels, J. Lange, T. Schäpers, and H. Lüth, Experimental and theoretical approach to spin splitting in modulation-doped $\mathrm{In}_{x} \mathrm{Ga}_{1-x} \mathrm{As} / \mathrm{InP}$ quantum wells for $B \rightarrow 0$, Phys. Rev. B 55, R1958(R) (1997).

[7] G. Schmidt, D. Ferrand, L. W. Molenkamp, A. T. Filip, and B. J. van Wees, Fundamental obstacle for electrical spin injection from a ferromagnetic metal into a diffusive semiconductor, Phys. Rev. B 62, R4790(R) (2000).

[8] E. I. Rashba, Theory of electrical spin injection: Tunnel contacts as a solution of the conductivity mismatch problem, Phys. Rev. B 62, R16267(R) (2000).

[9] A. Fert and H. Jaffrès, Conditions for efficient spin injection from a ferromagnetic metal into a semiconductor, Phys. Rev. B 64, 184420 (2001).

[10] H. C. Koo, J. H. Kwon, J. Eom, J. Chang, S. H. Han, and M. Johnson, Control of spin precession in a spin-injected field effect transistor, Science 325, 1515 (2009).

[11] F. J. Jedema, H. B. Heersche, A. T. Filip, J. J. A. Baselmans, and B. J. van Wees, Electrical detection of spin precession in a metallic mesoscopic spin valve, Nature (London) 416, 713 (2002). which may lead to instabilities and hysteretic curves in double-barrier systems [65]. Furthermore, magnetically doped resonant tunneling devices are shown to be quite sensitive to external magnetic fields [66-68]. In the presence of a spin-orbit coupling, beating patterns are predicted to occur in double-barrier resonant tunneling structures [69]. Finally, we would like to mention the closely related systems known as chaotic dots [70] since they are built as semiconductor cavities between a pair of quantum point contacts, similar to the two-dimensional cavities considered in the last part of our work. In contrast, our cavities have a regular shape. Interestingly, closed chaotic dots exhibit Coulomb blockade peak fluctuations [71], and subsequent discussions might then consider how these fluctuations are affected by the presence of spin-orbit interactions.

\section{ACKNOWLEDGMENT}

This work was funded by MINECO (Spain), Grant No. FIS2014-52564.

[12] F. Mireles and G. Kirczenow, Ballistic spin-polarized transport and Rashba spin precession in semiconductor nanowires, Phys. Rev. B 64, 024426 (2001).

[13] J.-S. Jeong and H.-W. Lee, Ballistic spin field-effect transistors: Multichannel effects, Phys. Rev. B 74, 195311 (2006).

[14] M. M. Gelabert, L. Serra, D. Sánchez, and R. López, Multichannel effects in Rashba quantum wires, Phys. Rev. B 81, 165317 (2010).

[15] J. C. Egues, G. Burkard, and D. Loss, Datta-Das transistor with enhanced spin control, Appl. Phys. Lett. 82, 2658 (2003).

[16] E. Y. Sherman and J. Sinova, Physical limits of the ballistic and nonballistic spin-field-effect transistor: Spin dynamics in remote-doped structures, Phys. Rev. B 72, 075318 (2005).

[17] B. K. Nikolić and S. Souma, Decoherence of transported spin in multichannel spin-orbit-coupled spintronic devices: Scattering approach to spin-density matrix from the ballistic to the localized regime, Phys. Rev. B 71, 195328 (2005).

[18] L. Xu, X.-Q. Li, and Q.-f. Sun, Revisit the spin-FET: Multiple reflection, inelastic scattering, and lateral size effects, Sci. Rep. 4, 7527 (2014).

[19] B. Y. Sun, P. Zhang, and M. W. Wu, Voltage-controlled spin precession in InAs quantum wells, Semicond. Sci. Technol. 26, 075005 (2011).

[20] P. Wójcik, J. Adamowski, B. J. Spisak, and M. Wołoszyn, Spin transistor operation driven by the Rashba spin-orbit coupling in the gated nanowire, J. Appl. Phys. 115, 104310 (2014).

[21] M. G. Pala, M. Governale, J. König, and U. Zülicke, Universal Rashba spin precession of two-dimensional electrons and holes, Europhys. Lett. 65, 850 (2004).

[22] P. Agnihotri and S. Bandyopadhyay, Analysis of the twodimensional Datta-Das spin field effect transistor, Phys. E (Amsterdam, Neth.) 42, 1736 (2010).

[23] A. N. M. Zainuddin, S. Hong, L. Siddiqui, S. Srinivasan, and S. Datta, Voltage-controlled spin precession, Phys. Rev. B 84, 165306 (2011). 
[24] M. M. Gelabert and L. Serra, Conductance oscillations of a spin-orbit stripe with polarized contacts, Eur. Phys. J. B 79, 341 (2011).

[25] M. I. Alomar, L. Serra, and D. Sánchez, Seebeck effects in two-dimensional spin transistors, Phys. Rev. B 91, 075418 (2015).

[26] P. Chuang, S. Ho, L. Smith, F. Sfigakis, M. Pepper, C. Chen, J. Fan, J. Griffiths, I. Farrer, H. Beere et al., All-electric allsemiconductor spin field-effect transistors, Nat. Nanotechnol. 10, 35 (2015).

[27] P. Debray, S. M. S. Rahman, J. Wan, R. S. Newrock, M. Cahay, A. T. Ngo, S. E. Ulloa, S. T. Herbert, M. Muhammad, and M. Johnson, All-electric quantum point contact spin-polarizer, Nat. Nanotechnol. 4, 759 (2009).

[28] M. P. Nowak and B. Szafran, Spin Current Source Based on a Quantum Point Contact with Local Spin-Orbit Interaction, Appl. Phys. Lett. 103, 202404 (2013).

[29] J. Schliemann, J. C. Egues, and D. Loss, Nonballistic SpinField-Effect Transistor, Phys. Rev. Lett. 90, 146801 (2003).

[30] K. C. Hall, W. H. Lau, K. Gündoğdu, M. E. Flatté, and T. F. Boggess, Nonmagnetic Semiconductor Spin Transistor, Appl. Phys. Lett. 83, 2937 (2003).

[31] B. Wang, J. Wang, and H. Guo, Quantum spin field effect transistor, Phys. Rev. B 67, 092408 (2003).

[32] D. Awschalom and N. Samarth, Spintronics without magnetism, Physics 2, 50 (2009).

[33] J. Wunderlich, B.-G. Park, A. C. Irvine, L. P. Zârbo, E. Rozkotová, P. Nemec, V. Novák, J. Sinova, and T. Jungwirth, Spin Hall effect transistor, Science 330, 1801 (2010).

[34] J.-F. Liu, K. S. Chan, and J. Wang, Nonmagnetic spin-field-effect transistor, Appl. Phys. Lett. 101, 082407 (2012).

[35] L. Serra, D. Sánchez, and R. López, Evanescent states in quantum wires with Rashba spin-orbit coupling, Phys. Rev. B 76, 045339 (2007).

[36] V. A. Sablikov and Y. Y. Tkach, Evanescent states in twodimensional electron systems with spin-orbit interaction and spin-dependent transmission through a barrier, Phys. Rev. B 76, 245321 (2007).

[37] A. Voskoboynikov, S. S. Liu, and C. P. Lee, Spin-dependent tunneling in double-barrier semiconductor heterostructures, Phys. Rev. B 59, 12514 (1999).

[38] E. A. de Andrada e Silva and G. C. La Rocca, Electron-spin polarization by resonant tunneling, Phys. Rev. B 59, R15583(R) (1999).

[39] T. Koga, J. Nitta, H. Takayanagi, and S. Datta, Spin-Filter Device Based on the Rashba Effect Using a Nonmagnetic Resonant Tunneling Diode, Phys. Rev. Lett. 88, 126601 (2002).

[40] D. Z.-Y. Ting and X. Cartoixà, Resonant interband tunneling spin filter, Appl. Phys. Lett. 81, 4198 (2002).

[41] M. M. Glazov, P. S. Alekseev, M. A. Odnoblyudov, V. M. Chistyakov, S. A. Tarasenko, and I. N. Yassievich, Spindependent resonant tunneling in symmetrical double-barrier structures, Phys. Rev. B 71, 155313 (2005).

[42] G. Isić, D. Indjin, V. Milanović, J. Radovanović, Z. Ikonić, and P. Harrison, Phase-breaking effects in double-barrier resonant tunneling diodes with spin-orbit interaction, J. Appl. Phys. 108, 044506 (2010).

[43] R. S. Calsaverini, E. Bernardes, J. C. Egues, and D. Loss, Intersubband-induced spin-orbit interaction in quantum wells, Phys. Rev. B 78, 155313 (2008).
[44] F. Dettwiler, J. Fu, S. Mack, P. J. Weigele, J. C. Egues, D. D. Awschalom, and D. M. Zumbühl, Electrical spin protection and manipulation via gate-locked spin-orbit fields, arXiv:1403.3518.

[45] S. M. Frolov, S. Luscher, W. Yu, Y. Ren, J. A. Folk, and W. Wegscheider, Ballistic spin resonance, Nature (London) 458, 868 (2009).

[46] M. O. Hachiya, G. Usaj, and J. C. Egues, Ballistic spin resonance in multisubband quantum wires, Phys. Rev. B 89, 125310 (2014).

[47] L. W. Molenkamp, G. Schmidt, and G. E. W. Bauer, Rashba Hamiltonian and electron transport, Phys. Rev. B 64, 121202 (2001).

[48] R. López, D. Sánchez, and L. Serra, From Coulomb blockade to the Kondo regime in a Rashba dot, Phys. Rev. B 76, 035307 (2007).

[49] E. Ott, Chaos in Dynamical Systems (Cambridge University Press, Cambridge, 1993).

[50] I. M. Ruzin, V. Chandrasekhar, E. I. Levin, and L. I. Glazman, Stochastic Coulomb blockade in a double-dot system, Phys. Rev. B 45, 13469 (1992).

[51] D. Sánchez, G. Platero, and L. L. Bonilla, Quasiperiodic current and strange attractors in ac-driven superlattices, Phys. Rev. B 63, 201306 (2001).

[52] D. Sánchez and L. Serra, Fano-Rashba effect in a quantum wire, Phys. Rev. B 74, 153313 (2006).

[53] I. A. Shelykh and N. G. Galkin, Fano and Breit-Wigner resonances in carrier transport through Datta and Das spin modulators, Phys. Rev. B 70, 205328 (2004).

[54] X. F. Wang, Spin transport of electrons through quantum wires with a spatially modulated Rashba spin-orbit interaction, Phys. Rev. B 69, 035302 (2004).

[55] L. Zhang, P. Brusheim, and H. Q. Xu, Multimode electron transport through quantum waveguides with spin-orbit interaction modulation: Applications of the scattering matrix formalism, Phys. Rev. B 72, 045347 (2005).

[56] S. Souma, A. Sawada, H. Chen, Y. Sekine, M. Eto, and T. Koga, Spin blocker using the interband Rashba effect in symmetric double quantum wells, Phys. Rev. Appl. 4, 034010 (2015).

[57] A. V. Moroz and C. H. W. Barnes, Effect of the spin-orbit interaction on the band structure and conductance of quasi-onedimensional systems, Phys. Rev. B 60, 14272 (1999).

[58] A. V. Moroz and C. H. W. Barnes, Spin-orbit interaction as a source of spectral and transport properties in quasi-onedimensional systems, Phys. Rev. B 61, R2464(R) (2000).

[59] C. S. Lent and D. J. Kirkner, The quantum transmitting boundary method, J. Appl. Phys. 67, 6353 (1990).

[60] C. Cohen-Tannoudji, B. Diu, and F. Laloë, Quantum Mechanics (Wiley-Interscience, New York, 1977), Vol. 1.

[61] L. Baskin, P. Neittaanmäki, B. Plamenevskii, and O. Sarafanov, Resonant Tunneling: Quantum Waveguides of Variable CrossSection, Asymptotics, Numerics, and Applications (Springer, Cham, Switzerland, 2015).

[62] X. H. Lou, C. Adelmann, S. A. Crooker, E. S. Garlid, J. Zhang, K. S. M. Reddy, S. D. Flexner, C. J. Palmstrom, and P. A. Crowel, Electrical detection of spin transport in lateral ferromagnetsemiconductor devices, Nat. Phys. 3, 197 (2007).

[63] A. D. Stone and P. A. Lee, Effect of Inelastic Processes on Resonant Tunneling in One Dimension, Phys. Rev. Lett. 54, 1196 (1985). 
[64] M. Buttiker, Coherent and sequential tunneling in series barriers, IBM J. Res. Dev. 32, 63 (1988).

[65] A. D. Martin, M. L. F. Lerch, P. E. Simmonds, and L. Eaves, Observation of intrinsic tristability in a resonant tunneling structure, Appl. Phys. Lett. 64, 1248 (1994).

[66] A. Slobodskyy, C. Gould, T. Slobodskyy, C. R. Becker, G. Schmidt, and L. W. Molenkamp, Voltage-Controlled Spin Selection in a Magnetic Resonant Tunneling Diode, Phys. Rev. Lett. 90, 246601 (2003).

[67] A. Slobodskyy, C. Gould, T. Slobodskyy, G. Schmidt, L. W. Molenkamp, and D. Sánchez, Resonant tunneling diode with spin polarized injector, Appl. Phys. Lett. 90, 122109 (2007).
[68] P. Wójcik, J. Adamowski, M. Wołoszyn, and B. J. Spisak, Spin filter effect at room temperature in $\mathrm{GaN} / \mathrm{GaMnN}$ ferromagnetic resonant tunneling diode, Appl. Phys. Lett. 102, 242411 (2013).

[69] J. C. Egues, C. Gould, G. Richter, and L. W. Molenkamp, Spin filtering and magnetoresistance in ballistic tunnel junctions, Phys. Rev. B 64, 195319 (2001).

[70] C. M. Marcus, A. J. Rimberg, R. M. Westervelt, P. F. Hopkins, and A. C. Gossard, Conductance Fluctuations and Chaotic Scattering in Ballistic Microstructures, Phys. Rev. Lett. 69, 506 (1992).

[71] I. Aleiner, P. Brouwer, and L. Glazman, Quantum effects in Coulomb blockade, Phys. Rep. 358, 309 (2002). 